\title{
TOPOLOGICALLY TRANSITIVE SEQUENCE OF COSINE OPERATORS ON ORLICZ SPACES
}

\author{
I. AKBARBAGLU*, M. R. AZIMI AND V. KUMAR
}

\begin{abstract}
For a Young function $\phi$ and a locally compact second countable group $G$, let $L^{\phi}(G)$ denote the Orlicz space on $G$. In this paper, we present a necessary and sufficient condition for the topological transitivity of a sequence of cosine operators $\left\{C_{n}\right\}_{n=1}^{\infty}:=$ $\left\{\frac{1}{2}\left(T_{g, w}^{n}+S_{g, w}^{n}\right)\right\}_{n=1}^{\infty}$, defined on $L^{\phi}(G)$. We investigate the conditions for a sequence of cosine operators to be topologically mixing. Further, we go on to prove a similar result for the direct sum of a sequence of cosine operators. Finally, we give an example of topologically transitive sequence of cosine operators.
\end{abstract}

\section{Introduction AND Preliminaries}

A sequence of bounded linear operators $\left\{T_{k}\right\}_{k \in \mathbb{N}_{0}}$ acting on a Fréchet space $X$ is said to be topologically transitive if for any pair $(U, V)$ consisting of two non-empty open subsets $U$ and $V$ of $X$, there exists an $n \in \mathbb{N}$ such that $T_{n}(U) \cap V \neq \emptyset$. A bounded linear operator $T$ is said to be topologically transitive if the sequence $\left\{S_{k}\right\}_{k \in \mathbb{N}_{0}}$ with $S_{k}:=T^{k}$, the $k$-iterates of $T$ with the convention that $T^{0}=I$, the identity operator, is topologically transitive as a sequence of bounded linear operators. A bounded linear operator $T$ is called hypercyclic if there exists a vector $x \in X$, called hypercyclic vector, such that the orbit $\left\{T^{k} x: k=0,1,2, \ldots\right\}$ of $x$ is dense in $X$, where $T^{0}$ is the identity operator on $X$. It is worth mentioning that these two notions, namely, topological transitivity and hypercyclicity of an operator are more likely equivalent on a Fréchet space $X[3,8]$. An operator $T$ is called topologically mixing whenever for any pair $(U, V)$ of two non-empty open subsets $U$ and $V$ of $X$, there exists an $N \in \mathbb{N}$ such that $T^{n}(U) \cap V \neq \emptyset$ for every $n \geq N$. An operator of the form $I+B$, where $B$ denotes the backward shift operator, is an example of topologically mixing operator which is also hypercyclic. We say a bounded linear operator $T$ on a Fréchet space $X$ is weakly mixing if and only if $T \oplus T$ is hypercyclic on $X \oplus X$. Note that weakly mixing operators are topologically transitive but the converse is not true in general. For more details on dynamics of linear operators, we refer the interested reader to two excellent books [3] and [8].

2010 Mathematics Subject Classification. Primary 47A16, 46E30 Secondary: 22D05.

Key words and phrases. Hypercyclicity; Topologically transitive; Topologically mixing; Weighted translation operator; Orlicz space; Locally compact groups.

${ }^{*}$ Corresponding author. 
A convex and even function $\phi: \mathbb{R} \rightarrow[0, \infty]$ with $\phi(0)=0$ is called a Young function if it possess one of the following conditions:

- $\phi$ is finite on $[0,+\infty)$ and is not identically to zero;

- There exists $x_{1}>0$ such that $\phi$ is finite on $\left[0, x_{1}\right]$ and $\phi(x)=+\infty$ for $x>x_{1}$;

- There exists $x_{1}>0$ such that $\phi$ is finite on $\left[0, x_{1}\right)$ and $\lim _{x \rightarrow x_{1}^{-}} \phi(x)=+\infty$ and $\phi(x)=+\infty$ for each $x \geq x_{1}$.

Corresponding to each Young function $\phi$, there is an associated Young function $\psi: \mathbb{R} \rightarrow$ $[0,+\infty]$ defined by $\psi(y):=\sup \{x|y|-\phi(x): x \geq 0\}$, which is called the complementary Young function of $\phi$. We remind that for a Young function $\phi$, its inverse i.e., $\phi^{-1}:[0,+\infty) \rightarrow[0,+\infty]$ is defined by $\phi^{-1}(y):=\inf \{x \geq 0: \phi(x)>y\}$ with $\inf (\emptyset)=+\infty$.

Let $G$ be a second countable locally compact group with the identity element $e$ and a right Haar measure $\lambda$. Let $L^{\phi}(G)$ denote the Orlicz space, consisting of all Borel measurable functions $f: G \rightarrow \mathbb{C}$ such that

$$
\int_{G} \phi(\alpha|f(x)|) d \lambda(x)<+\infty
$$

for some $\alpha>0$. It is well-known that the space $L^{\phi}(G)$ is a vector space. The Orlicz space $L^{\phi}(G)$ equipped with the Luxemburg-Nakano norm

$$
N_{\phi}(f)=\inf \left\{k>0: \int_{G} \phi\left(\frac{|f|}{k}\right) d \lambda \leq 1\right\},
$$

is a Banach space [11]. Moreover, if we assume that the Young function $\phi$ vanishes only at zero, then another equivalent norm called the Orlicz norm is defined on $L^{\phi}(G)$ by

$$
\|f\|_{\phi}:=\sup \left\{\int_{G}|f(x) \nu(x)| d \lambda(x): N_{\psi}(\nu) \leq 1\right\} .
$$

The set of all Borel functions $\nu$ on $G$ such that $N_{\psi}(\nu) \leq 1$ will be denoted by $\Omega$.

A Young function $\phi$ is said to satisfy $\Delta_{2}$-regular condition, if there is a constant $k>0$ such that $\phi(2 t) \leq k \phi(t)$ for large values of $t$ when $\lambda(G)<\infty$ and $\phi(2 t) \leq k \phi(t)$ for each $t>0$, whenever $\lambda(G)=\infty$. If $\phi$ is $\Delta_{2}$-regular, then the space $C_{c}(G)$ of all continuous functions on $G$ with compact support is dense in $L^{\phi}(G)$, and the dual space $\left(L^{\phi}(G),\|\cdot\|_{\phi}\right)$ is $\left(L^{\psi}(G), N_{\psi}(\cdot)\right)$. For further information, the interested reader is referred to [11].

It is well known that the hypercyclic phenomenon is occurred only on infinite-dimensional and separable spaces $[3,8]$. For this reason, we assume that $G$ is second countable and $\phi(x)=0$ if and only if $x=0$ [11, p. 87, Theorem 1]. Throughout this paper, the Banach space of all essentially bounded and measurable functions on $G$ is denoted by $L^{\infty}(G)$ and $N(f, r)$ denotes a neighborhood of $f \in L^{\phi}(G)$ with radius $r>0$. A bounded continuous function $w: G \rightarrow(0, \infty)$ is called a weight. For $g \in G$, let $\delta_{g}$ be the unit point mass at $g$. 
Given a weight $w$ on $G$ and $g \in G$, a weighted translation operator $T_{g, w}: L^{\phi}(G) \rightarrow L^{\phi}(G)$ is defined by

$$
T_{g, w}(f):=w \cdot f * \delta_{g}, \quad f \in L^{\phi}(G)
$$

where $f * \delta_{g}$ is the convolution defined as

$$
f * \delta_{g}(t):=\int_{G} f\left(t x^{-1}\right) d \delta_{g}(x)=f\left(t g^{-1}\right), \quad t \in G .
$$

Indeed, it is the right translation of $f$ by $g^{-1}$. Moreover, it is easy to check that $f * \delta_{g} \in L^{\phi}(G)$ whenever $f \in L^{\phi}(G)$. Recall that an element $g \in G$ is called a torsion element if it is of finite order. An element $g \in G$ is called periodic if the closed subgroup $G(g)$ generated by $g$ is compact. Further, an element in $G$ is aperiodic if it is not periodic. Equivalently, $g \in G$ is an aperiodic element, if and only if for any compact subset $K \subset G$, there exists an $N \in \mathbb{N}$ such that $K \cap K g^{-n}=\emptyset$ for $n>N$ [5, Lemma 2.1]. It is worth noting that a weighted translation $T_{g, w}$ cannot be hypercyclic whenever $\|w\|_{\infty} \leq 1$ or $g$ is a torsion element $[2,5]$. The hypercyclic weighted translations on locally compact groups was characterized by C. Chen [5] in details. In addition, he studied the hypercyclicity of weighted convolution operators.

Moreover, a study of the hypercyclic weighted translations on Orlicz spaces $L^{\phi}(G)$ was carried over by first two authors in [2].

If $w^{-1}:=\frac{1}{w} \in L^{\infty}(G)$, the weighted translation operator $T_{g, w}$ is invertible and its inverse is $T_{g^{-1}, w^{-1} * \delta_{g}}$ which will be denoted by $S_{g, w}$ throughout this paper. For each $n \in \mathbb{Z}$, the cosine operator $C_{n}: L^{\phi}(G) \rightarrow L^{\phi}(G)$ is defined by

$$
C_{n}:=\frac{1}{2}\left(T_{g, w}^{n}+S_{g, w}^{n}\right) .
$$

It follows that $2 C_{n} C_{m}=C_{n+m}+C_{n-m}$ for $n, m \in \mathbb{Z}$.

The study of cosine operator on Banach spaces is originally due to Bonilla and Miana [4]. They gave sufficient conditions for the hypercyclicity and topologically mixing of a strongly continuous cosine operator function. Afterwards, T. Kalmes in [9] characterized the hypercyclicity of cosine operator functions on $L^{p}(\Omega)\left(\Omega\right.$ is open subset of $\left.\mathbb{R}^{d}\right)$ generated by second order partial differential operators. He also showed that the hypercyclicity and weakly mixing of these type of operators are equivalent.

Furthermore, Chen [6] gave a necessary and sufficient condition for the topological transitivity of the cosine operator $C_{n}$ on $L^{p}(G)$. In this article, we study the topological transitivity of the cosine operator $C_{n}$ on a space which is a natural generalization of $L^{p}(G)$, namely, Orlicz space $L^{\phi}(G)$.

\section{Topological transitivity of $\left(C_{n}\right)_{n \in \mathbb{N}}$}

In this section we present our main results with some immediate consequences. We begin with the following theorem which give a necessary and sufficient condition on weight so that 
cosine operator is topologically transitive on a second countable locally compact group $G$ equipped with a right Haar measure $\lambda$.

Theorem 2.1. Let $g \in G$ be an aperiodic element of $G$ and let $\phi$ be a $\Delta_{2}$-regular Young function. Let $w, w^{-1} \in L^{\infty}(G)$. If $C_{n}:=\frac{1}{2}\left(T_{g, w}^{n}+S_{g, w}^{n}\right)$ is a sequence of cosine operators on $L^{\phi}(G)$, then the following statements are equivalent.

(i) $\left(C_{n}\right)_{n \in \mathbb{N}}$ is topologically transitive.

(ii) For each non-empty compact subset $K \subset G$ with $\lambda(K)>0$, there exist sequences of Borel sets $\left(E_{k}\right),\left(E_{k}^{+}\right)$and $\left(E_{k}^{-}\right)$in $K$, and a sequence $\left(n_{k}\right)$ of positive numbers such that for $E_{k}=E_{k}^{+} \cup E_{k}^{-}$, we have

$$
\lim _{k \rightarrow \infty} \sup _{\nu \in \Omega} \int_{K \backslash E_{k}}|\nu(x)| d \lambda(x)=0 .
$$

Moreover, the two sequence

$$
\varphi_{n}=\prod_{j=1}^{n} w * \delta_{g^{-1}}^{j} \quad \text { and } \quad \tilde{\varphi}_{n}=\left(\prod_{j=0}^{n-1} w * \delta_{g}^{j}\right)^{-1}
$$

satisfy

$$
\begin{aligned}
& \lim _{k \rightarrow \infty} \sup _{\nu \in \Omega} \int_{E_{k}} \varphi_{n_{k}}(x)\left|\nu\left(x g^{n_{k}}\right)\right| d \lambda(x)=0, \\
& \lim _{k \rightarrow \infty} \sup _{\nu \in \Omega} \int_{E_{k}} \tilde{\varphi}_{n_{k}}(x)\left|\nu\left(x g^{n_{k}}\right)\right| d \lambda(x)=0, \\
& \lim _{k \rightarrow \infty} \sup _{\nu \in \Omega} \int_{E_{k}^{+}} \varphi_{2 n_{k}}(x)\left|\nu\left(x g^{2 n_{k}}\right)\right| d \lambda(x)=0, \\
& \lim _{k \rightarrow \infty} \sup _{\nu \in \Omega} \int_{E_{k}^{-}} \tilde{\varphi}_{2 n_{k}}(x)\left|\nu\left(x g^{2 n_{k}}\right)\right| d \lambda(x)=0 .
\end{aligned}
$$

Proof. (i) $\Rightarrow$ (ii). In spite of being different underlying spaces, the approach of the proof is followed like as done in [6]. Let $K$ be a compact subset of $G$ such that $\lambda(K)>0$. Since $g \in G$ is an aperiodic element, there exists $N \in \mathbb{N}$ such that $K \cap K g^{ \pm n}=\emptyset$ for $n>N[5$, Lemma 2.1]. Denote the characteristic function of $K$ defined on $G$ by $\chi_{K}$. Clearly $\chi_{K} \in L^{\phi}(G)$. Take $\epsilon \in(0,1)$ and $U=N\left(\chi_{K}, \epsilon^{2}\right)$ and $V=N\left(-\chi_{K}, \epsilon^{2}\right)$ in the definition of the topologically transitive for the sequence $\left(C_{n}\right)_{n \in \mathbb{N}}$. There exist $f \in L^{\phi}(G)$ and $m \in \mathbb{N}$, of course, $m>N$ such that

$$
\left\|f-\chi_{K}\right\|_{\phi}<\epsilon^{2} \text { and }\left\|C_{m} f+\chi_{K}\right\|_{\phi}<\epsilon^{2} .
$$

Hence, we can write that

$$
\left\|\operatorname{Re}(f)-\chi_{K}\right\|_{\phi}<\epsilon^{2} \text { and }\left\|\operatorname{Re}\left(C_{m} f\right)+\chi_{K}\right\|_{\phi}=\left\|C_{m} \operatorname{Re}(f)+\chi_{K}\right\|_{\phi}<\epsilon^{2},
$$

where $\operatorname{Re}(f)$ is the real part of the complex valued function $f$. Since the map $\operatorname{Re}: L^{\phi}(G, \mathbb{C}) \rightarrow$ $L^{\phi}(G, \mathbb{R})$ is continuous and also commute with both $T_{g, w}$ and $S_{g, w}$, hence without loss of 
generality we may assume that the function $f$ is real valued. Therefore, for any Borel subset $F \subset G$, we have

$$
\begin{aligned}
\left\|C_{m} f^{+} \chi_{F}\right\|_{\phi} & \leq\left\|\left(C_{m} f^{+}\right)\right\|_{\phi}=\left\|\left(C_{m} f+\chi_{K}-\chi_{K}\right)^{+}\right\|_{\phi} \\
& \leq\left\|\left(C_{m} f+\chi_{K}\right)^{+}\right\|_{\phi}+\left\|\left(-\chi_{K}\right)^{+}\right\|_{\phi} \\
& =\left\|\left(C_{m} f+\chi_{K}\right)^{+}\right\|_{\phi} \leq\left\|C_{m} f+\chi_{K}\right\|_{\phi}<\epsilon^{2},
\end{aligned}
$$

where $f^{+}:=\max \{0, f\}$ and $f^{-}:=f^{+}-f$. Set $A=\{x \in K:|f(x)-1| \geq \epsilon\}$. Then

$$
\begin{aligned}
\epsilon^{2}>\left\|f-\chi_{K}\right\|_{\phi} & =\sup _{\nu \in \Omega} \int_{G}\left|f(x)-\chi_{K}(x)\right||\nu(x)| d \lambda(x) \\
& \geq \sup _{\nu \in \Omega} \int_{K}|f(x)-1||\nu(x)| d \lambda(x) \\
& \geq \epsilon \sup _{\nu \in \Omega} \int_{A}|\nu(x)| d \lambda(x) .
\end{aligned}
$$

Therefore, we have

$$
\sup _{\nu \in \Omega} \int_{A}|\nu(x)| d \lambda(x)<\epsilon
$$

Set $B_{m}=\left\{x \in K:\left|C_{m} f(x)+1\right| \geq \epsilon\right\}$. Then, by the similar argument, we get

$$
\sup _{\nu \in \Omega} \int_{B_{m}}|\nu(x)| d \lambda(x)<\epsilon
$$

Now, let $E_{m}:=\{x \in K:|f(x)-1|<\epsilon\} \cap\left\{x \in K:\left|C_{m} f(x)+1\right|<\epsilon\right\}$. Then, for $x \in E_{m}$, we get $f(x)>1-\epsilon>0$ and $C_{m} f(x)<\epsilon-1<0$. Also,

$$
\begin{aligned}
\sup _{\nu \in \Omega} \int_{K \backslash E_{m}}|\nu(x)| d \lambda(x) & =\sup _{\nu \in \Omega} \int_{A \cup B_{m}}|\nu(x)| d \lambda(x) \\
& =\sup _{\nu \in \Omega} \int_{A}|\nu(x)| d \lambda(x)+\sup _{\nu \in \Omega} \int_{B_{m}}|\nu(x)| d \lambda(x) \\
& <\epsilon+\epsilon=2 \epsilon .
\end{aligned}
$$


By keeping the facts that Haar measure $\lambda$ is right invariant, $T_{g, w}^{m} f^{+}$and $S_{g, w}^{m} f^{+}$are positive in the mind, with the aid of (2.1) we get that

$$
\begin{aligned}
2 \epsilon^{2} & > \\
= & \sup _{\nu \in \Omega} \int_{E_{m} g^{m}}\left|T_{g, w}^{m} f^{+}(x) \| \nu(x)\right| d \lambda(x) \\
= & \sup _{\nu \in \Omega} \int_{E_{m} g^{m}}\left|w(x) w\left(x g^{-1}\right) \ldots w\left(x g^{-m+1}\right) f^{+}\left(x g^{-m}\right) \| \nu(x)\right| d \lambda(x) \\
= & \sup _{\nu \in \Omega} \int_{E_{m}} w\left(x g^{m}\right) w\left(x g^{m-1}\right) \ldots w(x g) f^{+}(x)\left|\nu\left(x g^{m}\right)\right| d \lambda(x) \\
= & \sup _{\nu \in \Omega} \int_{E_{m}} \varphi_{m}(x) f^{+}(x)\left|\nu\left(x g^{m}\right)\right| d \lambda(x) \\
> & \sup _{\nu \in \Omega} \int_{E_{m}}(1-\epsilon) \varphi_{m}(x)\left|\nu\left(x g^{m}\right)\right| d \lambda(x) .
\end{aligned}
$$

Therefore,

$$
\sup _{\nu \in \Omega} \int_{E_{m}} \varphi_{m}(x)\left|\nu\left(x g^{m}\right)\right| d \lambda(x)<\frac{2 \epsilon^{2}}{1-\epsilon} .
$$

By the similar argument, we get

$$
2 \epsilon^{2}>\left\|\left(S_{g, w}^{m} f^{+}\right) \chi_{E_{m} g^{m}}\right\|_{\phi}>(1-\epsilon) \sup _{\nu \in \Omega} \int_{E_{m}} \tilde{\varphi}_{m}(x)\left|\nu\left(x g^{m}\right)\right| d \lambda(x)
$$

and thus,

$$
\sup _{\nu \in \Omega} \int_{E_{m}} \tilde{\varphi}_{m}(x)\left|\nu\left(x g^{m}\right)\right| d \lambda(x)<\frac{2 \epsilon^{2}}{1-\epsilon} .
$$

Hence, the first part of Condition (ii) holds as $\epsilon$ is arbitrary.

Now, let $E_{m}^{-}=\left\{x \in E_{m}: T_{g, w}^{m} f(x)<\epsilon-1\right\}$ and $E_{m}^{+}=E_{m} \backslash E_{m}^{-}$. Then, for $x \in E_{m}^{+}$, we have

$$
\epsilon-1>C_{m} f(x)=\frac{1}{2} T_{g, w}^{m} f(x)+\frac{1}{2} S_{g, w}^{m} f(x) \geq \frac{1}{2}(\epsilon-1)+\frac{1}{2} S_{g, w}^{m} f(x)
$$

and therefore,

$$
S_{g, w}^{m} f(x)<\epsilon-1, \quad x \in E_{m}^{+} .
$$


Now, consider the following

$$
\begin{aligned}
& (1-\epsilon) \sup _{\nu \in \Omega} \int_{E_{m}^{+}} \varphi_{2 m}(x)\left|\nu\left(x g^{2 m}\right)\right| d \lambda(x) \\
& <\sup _{\nu \in \Omega} \int_{E_{m}^{+}}\left|w\left(x g^{2 m}\right) w\left(x g^{2 m-1}\right) w\left(x g^{2 m-2}\right) \ldots w(x g)\right|\left|S_{g, w}^{m} f^{-}(x)\right|\left|\nu\left(x g^{2 m}\right)\right| d \lambda(x) \\
& =\sup _{\nu \in \Omega} \int_{E_{m}^{+} g^{2 m}}\left|w(x) w(x g) w\left(x g^{2}\right) \ldots w\left(x g^{-(2 m-1)}\right)\right|\left|S_{g, w}^{m} f^{-}\left(x g^{-2 m}\right)\right||\nu(x)| d \lambda(x) \\
& =\sup _{\nu \in \Omega} \int_{E_{m}^{+} g^{2 m}}\left|T_{g, w}^{2 m} S_{g, w}^{m} f^{-}(x)\right||\nu(x)| d \lambda(x) \\
& =\sup _{\nu \in \Omega} \int_{E_{m}^{+} g^{2 m}}\left|T_{g, w}^{m} f^{-}(x)\right||\nu(x)| d \lambda(x) \\
& \leq 2 \sup _{\nu \in \Omega} \int_{E_{m}^{+} g^{2 m}}\left|C_{m} f^{-}(x)\right||\nu(x)| d \lambda(x) \\
& =2 \sup _{\nu \in \Omega} \int_{G}\left|C_{m} f^{-}(x) \chi_{E_{m}^{+} g^{2 m}}\right||\nu(x)| d \lambda(x) \\
& =2 \sup _{\nu \in \Omega} \int_{G}\left|C_{m}\left(f^{+}-f\right)(x) \chi_{E_{m}^{+} g^{2 m}}\right||\nu(x)| d \lambda(x) \\
& =2 \sup _{\nu \in \Omega} \int_{G}\left|\left(C_{m} f^{+}\right) \chi_{E_{m}^{+} g^{2 m}}-\left(C_{m} f+\chi_{K}\right) \chi_{E_{m}^{+} g^{2 m}}+\chi_{K \cap E_{m}^{+} g^{2 m}}\right||\nu(x)| d \lambda(x) \\
& \leq 2 \sup _{\nu \in \Omega} \int_{G}\left|\left(C_{m} f^{+}\right) \chi_{E_{m}^{+} g^{2 m}}\right||\nu(x)| d \lambda(x) \\
& +2 \sup _{\nu \in \Omega} \int_{G}\left|\left(C_{m} f+\chi_{K}\right) \chi_{E_{m}^{+} g^{2 m}}\right||\nu(x)| d \lambda(x)+2 \sup _{\nu \in \Omega} \int_{K \cap E_{m}^{+} g^{2 m}}|\nu(x)| d \lambda(x) \\
& \leq 2\left\|\left(C_{m} f^{+} \chi_{E_{m}^{+} g^{2 m}}\right)\right\|_{\phi}+2\left\|\left(C_{m} f+\chi_{K}\right)\right\|_{\phi}+2\left\|\chi_{K \cap E_{m}^{+} g^{2 m}}\right\|_{\phi} \\
& <2 \epsilon^{2}+2 \epsilon^{2}+0=4 \epsilon^{2} \text {. }
\end{aligned}
$$

In the last inequality, from the fact $K \cap K g^{ \pm 2 m}=\emptyset$, has been already used. Therefore, we get

$$
\sup _{\nu \in \Omega} \int_{E_{m}^{+}} \varphi_{2 m}(x)\left|\nu\left(x g^{2 m}\right)\right| d \lambda(x)<\frac{4 \epsilon^{2}}{(1-\epsilon)} .
$$

In similar lines, we also have

$$
\sup _{\nu \in \Omega} \int_{E_{m}^{-}} \tilde{\varphi}_{2 m}(x)\left|\nu\left(x g^{2 m}\right)\right| d \lambda(x)<\frac{4 \epsilon^{2}}{(1-\epsilon)} .
$$

Since $\epsilon$ is arbitrary, last two condition of (ii) part also fulfilled.

(ii) $\Rightarrow$ (i). Let $U$ and $V$ be two non-empty open subsets of $L^{\phi}(G)$. Since $\phi$ is $\Delta_{2}$-regular we can choose two non-zero functions $f$ and $h$ in $C_{c}(G)$ such that $f \in U$ and $h \in V$. Set $K=\operatorname{supp}(f) \cup \operatorname{supp}(h)$, the supports of $f$ and $h$ respectively. Let $E_{k} \subset K$ and it satisfies condition (ii). But $g \in G$ is an aperiodic element, hence there exists $M \in \mathbb{N}$ such that $K \cap K g^{ \pm n}=\emptyset$ for all $n>M$. Subsequently, for a given $\epsilon>0$, one can find $N \in \mathbb{N}$ such that 
for each $k>N, n_{k}>M$ and

$$
\|h\|_{\infty} \cdot \sup _{\nu \in \Omega} \int_{E_{k}} \varphi_{n_{k}}(x)\left|\nu\left(x g^{n_{k}}\right)\right| d \lambda(x)<\epsilon, \quad\|h\|_{\infty} \sup _{\nu \in \Omega} \int_{K \backslash E_{k}}|\nu(x)| d \lambda(x)<\epsilon .
$$

Now, we have

$$
\begin{aligned}
\left\|T_{g, w}^{n_{k}}\left(h \chi_{E_{k}}\right)\right\|_{\phi} & =\sup _{\nu \in \Omega} \int_{G}\left|T_{g, w}^{n_{k}}\left(h \chi_{E_{k}}\right)(x) \nu(x)\right| d \lambda(x) \\
& =\sup _{\nu \in \Omega} \int_{G}\left|w(x) w\left(x g^{-1}\right) \ldots w\left(x g^{-n_{k}+1}\right) h\left(x g^{-n_{k}}\right) \chi_{E_{k}}\left(x g^{-n_{k}}\right) \nu(x)\right| d \lambda(x) \\
& =\sup _{\nu \in \Omega} \int_{G}\left|w\left(x g^{n_{k}}\right) w\left(x g^{n_{k}-1}\right) \ldots w(x g) h(x) \chi_{E_{k}}(x) \nu(x)\right| d \lambda(x) \\
& \leq\|h\|_{\infty} \cdot \sup _{\nu \in \Omega} \int_{E_{k}} \varphi_{n_{k}}(x)\left|\nu\left(x g^{n_{k}}\right)\right| d \lambda(x)<\epsilon .
\end{aligned}
$$

Hence,

$$
\lim _{k \rightarrow \infty}\left\|T_{g, w}^{n_{k}}\left(h \chi_{E_{k}}\right)\right\|_{\phi}=0
$$

Also,

$$
\begin{aligned}
\left\|h-h \chi_{E_{k}}\right\|_{\phi} & =\sup _{\nu \in \Omega} \int_{G}\left|h(x)-h(x) \chi_{E_{k}}(x)\right||\nu(x)| d \lambda(x) \\
& =\sup _{\nu \in \Omega} \int_{G}\left|h(x) \chi_{K \backslash E_{k}}(x)\right||\nu(x)| d \lambda(x) \\
& =\int_{K \backslash E_{k}}|h(x)||\nu(x)| d \lambda(x) \\
& \leq\|h\|_{\infty} \int_{K \backslash E_{k}}|\nu(x)| d \lambda(x)<\epsilon .
\end{aligned}
$$

By similar argument, using the conditions given in (ii) we get

$$
\lim _{k \rightarrow \infty}\left\|S_{g, w}^{n_{k}}\left(h \chi_{E_{k}}\right)\right\|_{\phi}=0, \lim _{k \rightarrow \infty}\left\|S_{g, w}^{2 n_{k}}\left(h \chi_{E_{k}^{-}}\right)\right\|_{\phi}=\lim _{k \rightarrow \infty}\left\|T_{g, w}^{2 n_{k}}\left(h \chi_{E_{k}^{+}}\right)\right\|_{\phi}=0 .
$$

In addition, we also have

$$
\lim _{k \rightarrow \infty}\left\|S_{g, w}^{n_{k}}\left(f \chi_{E_{k}}\right)\right\|_{\phi}=\lim _{k \rightarrow \infty}\left\|T_{g, w}^{n_{k}}\left(f \chi_{E_{k}}\right)\right\|_{\phi}=0 .
$$

For each $k \in \mathbb{N}$, we set

$$
v_{k}=f \chi_{E_{k}}+2 T_{g, w}^{n_{k}}\left(h \chi_{E_{k}^{+}}\right)+2 S_{g, w}^{n_{k}}\left(h \chi_{E_{k}^{-}}\right) .
$$

In this stage, an application of the frequently used fact i.e., $K \cap K g^{\left(m_{1}-m_{2}\right) n_{k}}=\emptyset\left(m_{1}, m_{2} \in \mathbb{Z}\right.$ and $m_{1} \neq m_{2}$ ) with Minkowski inequality yield that

$$
\begin{aligned}
\left\|v_{k}-f\right\|_{\phi} & \leq\left\|f-f \chi_{E_{k}}\right\|_{\phi}+2\left\|T_{g, w}^{n_{k}}\left(h \chi_{E_{k}^{+}}\right)\right\|_{\phi}+2\left\|S_{g, w}^{n_{k}}\left(h \chi_{E_{k}^{-}}\right)\right\|_{\phi} \\
& \leq \frac{\|f\|_{\infty}}{\phi^{-1}\left(\frac{1}{\lambda\left(K \backslash E_{k}\right)}\right)}+2\left\|T_{g, w}^{n_{k}}\left(h \chi_{E_{k}^{+}}\right)\right\|_{\phi}+2\left\|S_{g, w}^{n_{k}}\left(h \chi_{E_{k}^{-}}\right)\right\|_{\phi}
\end{aligned}
$$


and

$$
\begin{aligned}
\left\|C_{n_{k}} v_{k}-h\right\|_{\phi} & \leq\left\|h-h \chi_{E_{k}}\right\|_{\phi}+\frac{1}{2}\left\|T_{g, w}^{n_{k}}\left(f \chi_{E_{k}}\right)\right\|_{\phi}+\frac{1}{2}\left\|S_{g, w}^{n_{k}}\left(f \chi_{E_{k}}\right)\right\|_{\phi} \\
& +\left\|T_{g, w}^{2 n_{k}}\left(h \chi_{E_{k}^{+}}\right)\right\|_{\phi}+\left\|S_{g, w}^{2 n_{k}}\left(h \chi_{E_{k}^{-}}\right)\right\|_{\phi} \\
& \leq \frac{\|h\|_{\infty}}{\phi^{-1}\left(\frac{1}{\lambda\left(K \backslash E_{k}\right)}\right)}+\frac{1}{2}\left\|T_{g, w}^{n_{k}}\left(f \chi_{E_{k}}\right)\right\|_{\phi}+\frac{1}{2}\left\|S_{g, w}^{n_{k}}\left(f \chi_{E_{k}}\right)\right\|_{\phi} \\
& +\left\|T_{g, w}^{2 n_{k}}\left(h \chi_{E_{k}^{+}}\right)\right\|_{\phi}+\left\|S_{g, w}^{n_{k}}\left(h \chi_{E_{k}^{-}}\right)\right\|_{\phi} .
\end{aligned}
$$

Therefore, we have $\lim _{k \rightarrow \infty}\left\|v_{k}-f\right\|_{\phi}=0$ and $\lim _{k \rightarrow \infty}\left\|C_{n_{k}} v_{k}-h\right\|_{\phi}=0$, since $\lambda(K \backslash$ $\left.E_{k}\right) \rightarrow 0$ and it is assumed that $\sup \{x: \phi(x)<+\infty\}=+\infty$. Eventually, $\lim _{k \rightarrow \infty} v_{k}=f$ and $\lim _{k \rightarrow \infty} C_{n_{k}} v_{n_{k}}=h$. So, $C_{n_{k}}(U) \cap V \neq \emptyset$ for some $k$. Hence, the sequence $\left(C_{n}\right)_{n \in \mathbb{N}}$ is topologically transitive.

The following corollary gives a characterization of topologically mixing property of cosine operators on Orlicz space $L^{\phi}(G)$. Since the proof is similar to above theorem therefore we will omit the proof.

Corollary 2.2. Let $g \in G$ be an aperiodic element of $G$ and let $\phi$ be a $\Delta_{2}$-regular Young function. Let $w, w^{-1} \in L^{\infty}(G)$. If $C_{n}:=\frac{1}{2}\left(T_{g, w}^{n}+S_{g, w}^{n}\right)$ is cosine operator on $L^{\phi}(G)$ then the following statements are equivalent.

(i) $\left(C_{n}\right)_{n \in \mathbb{N}}$ is topologically mixing.

(ii) For each non-empty compact subset $K \subset G$ with $\lambda(K)>0$, there exist sequences of Borel sets $\left(E_{n}\right)$ such that

$$
\lim _{n \rightarrow \infty} \sup _{\nu \in \Omega} \int_{K \backslash E_{n}}|\nu(x)| d \lambda(x)=0
$$

and the two sequence

$$
\varphi_{n}=\prod_{j=1}^{n} w * \delta_{g^{-1}}^{j} \quad \text { and } \quad \tilde{\varphi}_{n}=\left(\prod_{j=0}^{n-1} w * \delta_{g}^{j}\right)^{-1}
$$

satisfy

$$
\begin{aligned}
& \lim _{n \rightarrow \infty} \sup _{\nu \in \Omega} \int_{E_{n}} \varphi_{n}(x)\left|\nu\left(x g^{n}\right)\right| d \lambda(x)=0, \\
& \lim _{n \rightarrow \infty} \sup _{\nu \in \Omega} \int_{E_{n}} \tilde{\varphi}_{n}(x)\left|\nu\left(x g^{n}\right)\right| d \lambda(x)=0 .
\end{aligned}
$$

We formulate the discrete version of Theorem 2.1. If $G$ is discrete group with the counting measure as its Haar measure. Then the set $E_{k}$ is nothing but set $K$ itself. Therefore, we have the following result.

Corollary 2.3. Let $g \in G$ be a non-torsion element of $G$ and let $\phi$ be a $\Delta_{2}$-regular Young function. Let $w, w^{-1} \in L^{\infty}(G)$. If $C_{n}:=\frac{1}{2}\left(T_{g, w}^{n}+S_{g, w}^{n}\right)$ is cosine operator on $L^{\phi}(G)$ then the following statements are equivalent. 
(i) $\left(C_{n}\right)_{n \in \mathbb{N}}$ is topologically transitive.

(ii) For each non-empty finite subset $K \subset G$, there exist sequences of Borel sets $\left(E_{k}^{+}\right)$and $\left(E_{k}^{-}\right)$in $K$, and a sequence $\left(n_{k}\right)$ of positive numbers such that for $K=E_{k}^{+} \cup E_{k}^{-}$, we have the two sequence

$$
\varphi_{n}=\prod_{j=1}^{n} w * \delta_{g^{-1}}^{j} \quad \text { and } \quad \tilde{\varphi}_{n}=\left(\prod_{j=0}^{n-1} w * \delta_{g}^{j}\right)^{-1}
$$

satisfy

$$
\begin{gathered}
\lim _{k \rightarrow \infty} \sup _{\nu \in \Omega} \sum_{x \in K} \varphi_{n_{k}}(x)\left|\nu\left(x g^{n_{k}}\right)\right|=0, \quad \lim _{k \rightarrow \infty} \sup _{\nu \in \Omega} \sum_{x \in K} \tilde{\varphi}_{n_{k}}(x)\left|\nu\left(x g^{n_{k}}\right)\right|=0, \\
\lim _{k \rightarrow \infty} \sup _{\nu \in \Omega} \sum_{x \in E_{k}^{+}} \varphi_{2 n_{k}}(x)\left|\nu\left(x g^{2 n_{k}}\right)\right|=0, \text { and } \lim _{k \rightarrow \infty} \sup _{\nu \in \Omega} \sum_{x \in E_{k}^{-}} \tilde{\varphi}_{2 n_{k}}(x)\left|\nu\left(x g^{2 n_{k}}\right)\right|=0 .
\end{gathered}
$$

Here we present a characterization of topological transitivity for a finite sequence of weighted cosine operators. We set the following notations for the sequence. For a fix $M \in \mathbb{N}$. Let $\left\{g_{l}\right\}_{1 \leq l \leq M}$ and $\left\{w_{l}\right\}_{1 \leq l \leq M}$ be the sequences of aperiodic elements of group $G$ and positive weights respectively. Then $\left\{T_{g_{l}, w_{l}}\right\}_{1 \leq l \leq M}$ is a sequence of weighted translation operators. We have the following characterization.

Theorem 2.4. Let $\left\{g_{l}\right\}_{l}$ and $\left\{w_{l}\right\}_{l}$ be the sequences of aperiodic elements and positive weights respectively such that $w_{l}, w_{l}^{-1} \in L^{\infty}(G)$. Let $C_{l, n}:=\frac{1}{2}\left(T_{g_{l}, w_{l}}^{n}+S_{g_{l}, w_{l}}^{n}\right)$ be the cosine operators on $L^{\phi}(G)$ for $1 \leq l \leq M$, where $T_{g_{l}, w_{l}}$ is the weighted translation operator. Then the following statements are equivalent.

(i) $\left(C_{1, n} \oplus C_{2, n} \oplus \cdots \oplus C_{M, n}\right)_{n \in \mathbb{N}_{0}}$ is topologically transitive.

(ii) For each non-empty compact subset $K \subset G$ with $\lambda(K)>0$, there is some sequence $\left(n_{k}\right)$ of positive integers such that for $1 \leq l \leq M$, there exist sequences of Borel sets $\left(E_{l, k}\right),\left(E_{l, k}^{+}\right)$and $\left(E_{l, k}^{-}\right)$such that for $E_{l, k}=E_{l, k}^{+} \cup E_{l, k}^{-}$, we have

$$
\lim _{k \rightarrow \infty} \sup _{\nu \in \Omega} \int_{K \backslash E_{l, k}}|\nu(x)| d \lambda(x)=0
$$

and the two sequence

$$
\varphi_{l, n_{k}}=\prod_{j=1}^{n_{k}} w_{l} * \delta_{g_{l}}^{j} \text { and } \tilde{\varphi}_{l, n_{k}}=\left(\prod_{j=0}^{n_{k}-1} w_{l} * \delta_{g_{l}}^{j}\right)^{-1}
$$

satisfy

$$
\begin{aligned}
& \lim _{k \rightarrow \infty} \sup _{\nu \in \Omega} \int_{E_{l, k}} \varphi_{l, n_{k}}(x)\left|\nu\left(x g^{n_{k}}\right)\right| d \lambda(x)=0, \\
& \lim _{k \rightarrow \infty} \sup _{\nu \in \Omega} \int_{E_{l, k}} \tilde{\varphi}_{l, n_{k}}(x)\left|\nu\left(x g^{n_{k}}\right)\right| d \lambda(x)=0,
\end{aligned}
$$




$$
\begin{aligned}
& \lim _{k \rightarrow \infty} \sup _{\nu \in \Omega} \int_{E_{l, k}^{+}} \varphi_{l, 2 n_{k}}(x)\left|\nu\left(x g^{2 n_{k}}\right)\right| d \lambda(x)=0, \\
& \lim _{k \rightarrow \infty} \sup _{\nu \in \Omega} \int_{E_{l, k}^{-}} \tilde{\varphi}_{l, 2 n_{k}}(x)\left|\nu\left(x g^{2 n_{k}}\right)\right| d \lambda(x)=0 .
\end{aligned}
$$

Proof. (i) $\Rightarrow$ (ii). Let $K$ be a compact subset of $G$ such that $\lambda(K)>0$. Since $\left(C_{1, n} \oplus C_{2, n} \oplus\right.$ $\left.\cdots \oplus C_{N, n}\right)_{n \in \mathbb{M}_{0}}$ is topologically transitive, for $\epsilon \in(0,1)$, there exist $f_{l} \in L^{\phi}(G)$ and $m \in N$ such that for $1 \leq l \leq M$, we have

$$
\left\|f_{l}-\chi_{K}\right\|_{\phi}<\epsilon^{2} \text { and }\left\|C_{l, m} f_{l}+\chi_{K}\right\|_{\phi}<\epsilon^{2} .
$$

Further, to complete the proof follow the proof of part (i) $\Rightarrow$ (ii) of Theorem 2.1 to get desired conditions on weights $w_{l}$ for each $l$.

(ii) $\Rightarrow$ (i). Let $U_{l}$ and $V_{l}$ be non-empty open subsets of $L^{\phi}(G)$. Since $\phi$ is $\Delta_{2}$-regular we can choose two non-zero functions $f_{l}$ and $h_{l}$ in $C_{c}(G)$ such that $f_{l} \in U_{l}$ and $h \in V_{l}$. Set $K=\operatorname{supp}\left(f_{l}\right) \cup \operatorname{supp}\left(h_{l}\right)$ (the supports of functions $f_{l}$ and $h_{l}$ ). Let $E_{l, k} \subset K$ and it satisfies condition (ii). Now, imitate the proof of (ii) $\Rightarrow$ (i) of Theorem 2.1 to get that $C_{l, n_{k}}\left(U_{l}\right) \cap V_{l} \neq \emptyset$ for each $l, 1 \leq l \leq M$.

Remark 2.1. $\quad$ (a) As elementary example of $\Delta_{2}$-regular Young function let $\phi(x)=x^{p} / p$ and $\psi(x)=x^{q} / q$ for $1<p, q<\infty$ with $1 / p+1 / q=1$. Also, let $\phi_{1}(x)=$ $|x|^{\alpha}(1+|\log | x||)$ with $\alpha>1$ and $\phi_{2}(x)=|x|^{\alpha} \ln ^{\beta}(|x|+e)$ with $\beta \geq 1$ and $\alpha>1$. By [11, Corollary 2.3.4] for $i=1,2, \phi_{i}$ and its complementary function, $\psi_{i}$, satisfy the $\Delta_{2}$-regular condition.

(b) Theorems 2.1 and 2.4 extends [6, Theorem 2.1] and [6, Corollary 2.7] from weighted Lebesgue spaces to weighted Orlicz spaces.

Example 2.1. Let $G=\mathbb{Z}$. Fix an aperiodic element $g \in \mathbb{Z}$ with $g \geq 1$. Define the Young function $\phi(x)=(1+|x|) \ln (1+|x|)-|x|$, and consider the weight function

$$
w(i)= \begin{cases}\frac{1}{2}, & i \geq 0, \\ \frac{3}{2}, & i<0 .\end{cases}
$$

A direct computation gives the complementary of Young function $\psi$ to $\phi$ which is given by $\psi(x)=\exp (|x|)-|x|-1$.

Note that $\phi$ is $\Delta_{2}$-regular and vanishes only at zero.

Choose an arbitrary $\nu \in \Omega$. Then, $\sum_{n=-\infty}^{+\infty} \psi(|\nu(n)|) \leq 1$ if and only if $\sum_{n=-\infty}^{+\infty}(\exp (|\nu(n)|)-$ $|\nu(n)|-1) \leq 1$. But the last is established only if $|\nu| \leq 2$. Each compact subset $K \subset \mathbb{Z}$ is a finite set, consisting of the integers $a_{1} \leq a_{2} \leq \cdots \leq a_{m}$. Take $E_{i}:=\left\{a_{1}, \cdots, a_{i}\right\}$ and for each $i \geq m$, define $E_{i}:=E_{m}$. Put $n_{k}=i, E_{k}^{+}:=E_{i}$ and $E_{k}^{-}:=\emptyset$ in the statement (ii) of 
Theorem 2.1. In this circumstances, we have

$$
\lim _{n \rightarrow \infty} \sup _{\nu \in \Omega} \sum_{j \in K \backslash E_{n}}|\nu(j)|=0 .
$$

In addition, one may find $n_{0} \in \mathbb{N}$ such that $a_{1}+n_{0} g \geq 0$. Hence, for each $n \geq n_{0}$ we have,

$$
\begin{gathered}
\sup _{\nu \in \Omega} \sum_{a_{i} \in E_{n}} \varphi_{n}\left(a_{i}\right)\left|\nu\left(a_{i}+n g\right)\right| \leq 2 \sum_{a_{i} \in E_{n}} \varphi_{n}\left(a_{i}\right) \\
=2 \sum_{a_{i} \in E_{n}} w\left(a_{i}+g\right) w\left(a_{i}+2 g\right) \cdots w\left(a_{i}+n g\right) \\
\leq 2 \sum_{a_{i} \in E_{n}} w\left(a_{1}+g\right) w\left(a_{1}+2 g\right) \cdots \\
w\left(a_{1}+n_{0} g\right) w\left(a_{1}+\left(n_{0}+1\right) g\right) \cdots w\left(a_{1}+n g\right) \\
\leq 2 \sum_{a_{i} \in E_{n}}\left(\frac{1}{2}\right)^{n-n_{0}} w\left(a_{1}\right)^{\left|a_{1}\right|} \\
=2 \operatorname{card}\left(E_{n}\right)\left(\frac{1}{2}\right)^{n-n_{0}} w\left(a_{1}\right)^{\left|a_{1}\right|} \\
\leq 2 m\left(\frac{1}{2}\right)^{n-n_{0}} w\left(a_{1}\right)^{\left|a_{1}\right|} \rightarrow 0
\end{gathered}
$$

as $n \rightarrow \infty$. Here, $\operatorname{card}\left(E_{n}\right)$ means the cardinality of the set $E_{n}$.

Similarly, there exists $t_{0} \in \mathbb{N}$ such that $a_{m}-t_{0} g \leq 0$ and so for each $n \geq t_{0}$,

$$
\begin{gathered}
\sup _{\nu \in \Omega} \sum_{a_{i} \in E_{n}} \tilde{\varphi}_{n}\left(a_{i}\right)\left|\nu\left(a_{i}+n g\right)\right| \leq 2 \sum_{a_{i} \in E_{n}} \tilde{\varphi}_{n}\left(a_{i}\right) \\
=2 \sum_{a_{i} \in E_{n}} w^{-1}\left(a_{i}-g\right) w^{-1}\left(a_{i}-2 g\right) \cdots w^{-1}\left(a_{i}-n g\right) \\
\leq 2 \sum_{a_{i} \in E_{n}} w^{-1}\left(a_{m}-g\right) w^{-1}\left(a_{m}-2 g\right) \cdots \\
w^{-1}\left(a_{m}-t_{0} g\right) w^{-1}\left(a_{m}-\left(t_{0}+1\right) g\right) \cdots w^{-1}\left(a_{m}-n g\right) \\
\leq 2 \sum_{a_{i} \in E_{n}}\left(\frac{2}{3}\right)^{n-t_{0}} w^{-1}\left(a_{m}\right)^{\left|a_{m}\right|} \\
\leq 2 m\left(\frac{2}{3}\right)^{n-t_{0}} w^{-1}\left(a_{m}\right)^{\left|a_{m}\right|} \rightarrow 0,
\end{gathered}
$$

as $n \rightarrow \infty$. The other statements of Theorem 2.1 can be verified in this way. Therefore, by Theorem 2.1, the corresponding sequence of cosine operators to the weight $w$ and $g$, is topologically transitive.

\section{ACKNOWLEDGMent}

Vishvesh Kumar is supported by FWO Odysseus 1 Grant G.0H94.18N: Analysis and Partial Differential Equations. We thank the referee for useful comments and remarks, which helped to improve the final version of the paper.

\section{REFERENCES}

[1] Shamim I. Ansari, Hypercyclic and cyclic vectors, J. Funct. Anal. 128 (1995), no. 2, 374-383.

[2] M. R. Azimi and I. Akbarbaglu, Hypercyclicity of weighted translations on Orlicz spaces, Oper. Matrices 12 (2018), no. 1, 27-37.

[3] Frédéric Bayart and Étienne Matheron, Dynamics of linear operators, Cambridge Tracts in Mathematics, Cambridge University Press, Cambridge, 2009. 
[4] Antonio Bonilla, Pedro J. Miana, Hypercyclic and topologically mixing cosine functions on Banach spaces, Proc. Am. Math. Soc. 136 (2008) 519528.

[5] C. Chen, C-H. Chu, Hypercyclic weighted translations on groups, Proc. Amer. Math. Soc. 139 (2011), no. 8, 2839-2846.

[6] Chung-Chuan Chen, Topological transitivity for cosine operator functions on groups, Topology Appl. 191 (2015), 48-57.

[7] Chung-Chuan Chen, Seyyed Mohammad Tabatabaie, Chaotic operators on hypergroups, Oper. Matrices 12 (2018), no. 1, 143-156.

[8] Karl-G. Grosse-Erdmann and Alfredo Peris Manguillot, Linear chaos, Universitext, Springer, London, 2011.

[9] T. Kalmes, Hypercyclicity and mixing for cosine operator functions generated by second order partial differential operators, J. Math. Anal. Appl. 365 (2010) 363375.

[10] C. Kitai, Invariant closed sets for linear opeartors, Thesis (Ph.D.)University of Toronto (Canada), 1982.

[11] M. M. Rao and Z. D. Ren, Theory of Orlicz spaces, Monographs and Textbooks in Pure and Applied Mathematics, 146. Marcel Dekker, Inc., New York, 1991.

[12] Héctor N. Salas, Hypercyclic weighted shifts, Trans. Amer. Math. Soc., 347 (3) (1995) 993-1004.

IBRAHIM AKBARBAGLU

Email address: ibrahim.akbarbaglu@gmail.com, i.akbarbaglu@cfu.ac.ir

Department of Mathematics, Farhangian University, Tehran, Iran.

Mohammad Reza Azimi

Email address: mhr.azimi@maragheh.ac.ir

Department of Mathematics, Faculty of Sciences, University of Maragheh, 55181-83111, MARAGHEH, IRAN.

VishVESh KumaR

Email address: vishveshmishra@gmail.com

Department of Mathematics: Analysis, Logic and Discrete Mathematics, Ghent University, Krijgslaan 281, Building S8, 9000, Ghent, Belgium 